\title{
Immunvermittelte und Autoimmun-Enteropathien
}

\author{
Philip M. Sherman ${ }^{a}$ Ernest Cutz $^{\mathrm{a}}$ Olivier Goulet $^{\mathrm{b}}$ \\ ${ }^{a}$ Hospital for Sick Children, Universität Toronto, Toronto, Ont., Kanada; ${ }^{b}$ Groupe Hospitalier Necker - \\ Enfants Malades, Paris, Frankreich
}

\section{Schlüsselwörter}

Diarrhoe, frühkindliche $\cdot$ Enteropathie, immunvermittelt und autoimmune - IPEX-Syndrom • Autoimmunität

\section{Zusammenfassung}

Die Autoimmun-Enteropathie manifestiert sich im Säuglingsalter normalerweise in Form einer protrahierten Diarrhoe. Zugrunde liegende Störungen der Immunfunktion, u.a. der regulatorischen T-Zellen, sind auszuschliessen. Zu den Behandlungsoptionen zählen Ernährungsrehabilitation, Immunsuppression und in besonderen Fällen eine Knochenmarktransplantation.

Copyright (c) 2006 Nestec Ltd., Vevey/S. Karger AG, Basel

\section{Einleitung}

Die Autoimmun-Enteropathie und Enteropathien aufgrund primärer Immundefekte zählen zu den relativ seltenen Ursachen einer frühkindlichen protrahierten Diarrhoe. Bei Säuglingen oder Kleinkindern mit chronischer unspezifischer Diarrhoe gestaltet sich die Differenzialdiagnose relativ umfassend [1], wobei andere Ursachen in Erwägung gezogen werden müssen als bei
Erwachsenen mit den gleichen Symptomen [2, 3]. In den meisten Ländern der Welt zählen infektiöse und postinfektiöse Enteropathien nach wie vor zu den häufigsten Ursachen der protrahierten Diarrhoe [4]. Einige Forscher behaupten, allergische Enteropathien seien bei Kleinkindern ebenfalls weit verbreitet, selbst wenn weitere atopische Symptome fehlen oder die familiäre Anamnese keinen Hinweis auf eine allergische Prädisposition enthält. Viel häufiger entwickelt sich bei Neugeborenen und Säuglingen, auch wenn diese ausschliesslich gestillt werden, eine durch Milch- und Sojaeiweiss induzierte allergische Colitis. Bei älteren Kindern, die bereits glutenhaltige Beikost erhalten, muss die Zöliakie als Ursache der Symptome in Betracht gezogen werden, da zunehmend klar wird, dass sich die glutensensitive Enteropathie auf viele verschiedene Arten und Weisen manifestiert und eine lebenslange Erkrankung ist, die durch eine entsprechende Ernährungsumstellung wirksam und sicher behandelt werden kann [5].

Hilfreich bei der Abgrenzung alternativer Ätiologien ist, dass die Ursachen der chronischen Diarrhoe, bei denen die Zotten-Krypten-Struktur relativ intakt bleibt, von den Ursachen abgrenzt werden, bei denen eine Veränderung der Zotten-Krypten-Struktur (d.h. eine Enteropathie) stattfindet. Im ersten Fall müssen Erkrankungen, wie z.B. angeborene Störungen des Ionentransports und

\section{KARGER}

Fax +4161306 1234

E-Mail karger@karger.ch

www.karger.com
C 2006 Nestec Ltd, Vevey/S. Karger AG, Basel 0250-9652/06/0641-0007\$23.50/0

Online-Zugriff auf: www.karger.com/and
Philip M. Sherman, MD, FRCPC

Gastroenterology and Nutrition, Room 8409

Hospital for Sick Children, 555 University Avenue

Toronto, Ont. M5G 1X8 (Kanada)

Tel. +1 416813 7734, Fax +1 416813 6531, E-Mail philip.sherman@sickkids.ca 
sekretorische Diarrhoe aufgrund bakterieller Enterotoxine bzw. Tumoren, die Hormone freisetzen (funktionell aktiv), in Betracht gezogen werden [1]. Demgegenüber sollte man bei einer Enteropathie mit Zottenatrophie an eine vollständig andere Gruppe möglicher Diagnosen denken. Nach Ausschluss infektiöser und allergischer Ätiologien sollte bei chronischen, stark voluminösen Durchfällen in den ersten Lebenstagen u.a. die Enteropathie mit Einschluss von Mikrovilli [6], die auch als kongenitale Mikrovillusatrophie [7] oder Davidson-Syndrom bezeichnet wird, in Betracht gezogen werden.

Setzt die chronische Diarrhoe etwas später, d.h. nicht in den ersten Lebenstagen, ein, so kann es sich um eine Tufting-Enteropathie [8] handeln, eine Erkrankung, die auch als Darmepithelzelldysplasie [9] bezeichnet wird und in ihrer Manifestation stark einer Autoimmun-Enteropathie ähnelt. Interessanterweise stammen bei den aus Europa und Nordamerika gemeldeten Fällen viele Säuglinge, die unter dieser Erkrankung leiden, aus Familien, die ursprünglich aus Nordafrika, der Türkei und insbesondere von der Insel Malta kommen. Bei Säuglingen dieser ethnischen Abstammung sollte die Tufting-Enteropathie daher stets ernsthaft in Erwägung gezogen werden.

\section{Klinische Präsentation}

Bei Kindern mit Autoimmun-Enteropathie äussert sich die Erkrankung in der Regel nach der Neugeborenenphase, jedoch innerhalb des ersten Lebensjahres. Lag bei der Mutter während der Schwangerschaft ein Polyhydramnion vor bzw. finden sich in der Familie weitere Kinder mit Autoimmun-Enteropathie, so sollte nach weiteren Ursachen der frühkindlichen protrahierten Diarrhoe geforscht werden, wie z.B. der kongenitalen Chloriddiarrhoe [1].

Die meisten Fälle von Autoimmun-Enteropathie beginnen im Alter zwischen zwei und vier Monaten nach einer normalen Schwangerschaft und Neugeborenenperiode mit chronischen, wässrigen, unablässigen Durchfällen. Es sind jedoch durchaus auch Fälle bekannt, bei denen die Symptome später einsetzten, und es gibt sogar Berichte über vereinzelte Fälle, bei denen sich die Durchfallerkrankung erstmals in der Adoleszenz [10] bzw. im Erwachsenenalter [11] manifestierte. Bei diesen Patienten, bei denen die Erkrankung erstmals in einem höheren Alter auftritt, lautet die Diagnose bis zum Nachweis von Enterozyten-Autoantikörpern in der Regel refraktäre Sprue. Ob die molekulare Basis der Erkrankung bei den Fällen, die sich erst in späteren Jahren manifestieren, die gleiche ist wie bei den Fällen, bei denen sich die Diarrhoe bereits im ersten Lebensjahr entwickelt, bleibt abzuklären.

Obwohl sich diese Autoimmunerkrankungen in erster Linie als Erkrankungen des Dünndarms äussern, ist der Dickdarm häufig ebenfalls betroffen. Bei Anzeichen und Symptomen einer Colitis (z.B. Tenesmen, Stuhldrang, schleimig-blutige Stuhlgänge), die mit einer protrahierten Diarrhoe einhergehen, welche auf einen Behandlungsversuch in Form einer Elementardiät nicht anspricht, sollte - insbesondere in Verbindung mit Wachstumshemmung und Mangelernährung - der Verdacht auf Autoimmun-Enteropathie berücksichtigt werden.

Auch das Vorliegen weiterer Autoimmunerkrankungen erhöht das Risiko einer Autoimmun-Enteropathie. Die ersten, in der wissenschaftlichen Literatur beschriebenen Fälle der Autoimmun-Enteropathie wurden mit Typ-1-Diabetes mellitus, Autoimmun-Thyroiditis, autoimmun-hämolytischer Anämie sowie dermatologischen Erkrankungen, die wahrscheinlich autoimmunen Ursprungs waren, in Zusammenhang gebracht. Viele der gemeldeten Fälle standen ferner in Zusammenhang mit Tubulusnephrose.

\section{Histopathologische Befunde}

Dünndarmbiopsien erweisen sich bei Säuglingen und Kindern mit protrahierter Diarrhoe als extrem hilfreich. Die Ergebnisse morphologischer und immunohistochemischer Untersuchungen sind nicht nur hilfreich, wenn es darum geht, eine bestimmte Diagnose zu stellen, sondern können auch oft bei nicht eindeutiger Bewertung andere Krankheiten ausschliessen [12].

Bei der Autoimmun-Enteropathie weisen die lichtmikroskopischen Befunde (Abb. 1a) häufig viele Merkmale auf, die mit der glutensensitiven Enteropathie in Verbindung gebracht werden, selbst wenn bei den betroffenen Kindern noch keine Exposition gegenüber Gluten stattgefunden hat. Untersucht man Schnittpräparate, die mit Hematoxylin-Eosin gefärbt werden, zeigt sich häufig eine totale Zottenatrophie und Kryptenhyperplasie. Ferner findet man häufig eine ausgeprägte Infiltration der Lamina propria der Darmschleimhaut durch Immunzellen, u.a. aktivierte T-Zellen [13]. Unter Umständen finden sich auch morphologische Nachweise intraepithelialer Lymphozyten, jedoch nicht in dem selben Ausmass wie bei Kindern, die unter einer durch Zöliakie verursachten totalen Zottenatrophie leiden [14]. Immunohistochemische Untersuchungen, bei denen relevante Antikörper als 
Abb. 1. a Biopsie der Dünndarmschleimhaut von einem Kind mit protrahierter Diarrhoe. Die Biopsie zeigt eine totale Zottenatrophie mit Kryptenhyperplasie sowie Infiltration der Lamina propria durch ein gemischtes, chronisch-entzündliches Zellinfiltrat. Hematoxylin-Eosin-Färbung. Ungefähr 20-fache Vergrösserung. b Die immunohistochemische Untersuchung mit Anti-CD3-Antikörpern zeigt eine Infiltration der Lamina propria durch zahlreiche CD3+ T-Zellen. Ungefähr 20-fache Vergrösserung.

Abb. 2. Mittels indirekter Immunfluoreszenz werden Enterozyten-Autoantikörper im Blutserum eines Kindes mit Autoimmun-Enteropathie nachgewiesen. Das Fluoreszenzmuster zeigt die Umrisse der apikalen Oberfläche der Enterozyten entlang der Zotten-Krypten-Struktur.
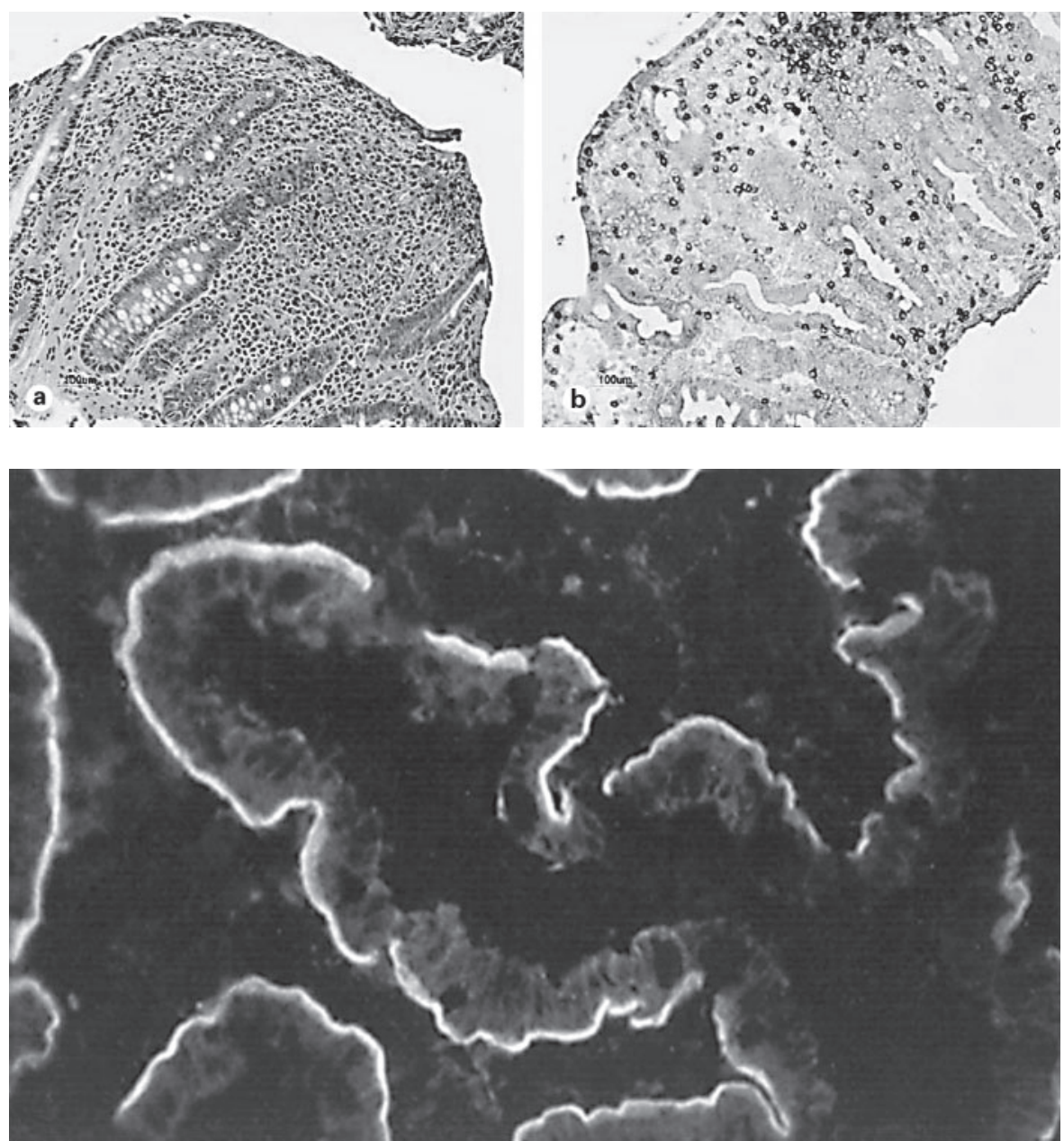

Marker eingesetzt werden, liefern den Nachweis einer ausgeprägten Infiltration der Lamina propria durch CD3+ T-Zellen (Abb. 1b). Neuere Erkenntnisse weisen darauf hin, dass das Zytokin Interleukin (IL)-21 möglicherweise die T-Zellen aktiviert, das Überleben der Zellen stört und letztendlich den betroffenen Wirtsorganismus für $\mathrm{Au}$ toimmunerkrankungen prädisponiert [15].

In den Dickdarmbiopsien von Kindern mit Autoimmun-Enteropathie finden sich ausserdem Belege für eine Infiltration der Lamina propria durch Immunzellen. Dieser Befund unterscheidet sich stark von den Befunden anderer Ursachen der frühkindlichen protrahierten Diarrhoe, bei denen keine Anzeichen einer Immunaktivierung ausserhalb des Dünndarms beobachtet werden [14].

\section{Autoantikörper}

Kennzeichnend für die Autoimmun-Enteropathie ist der Nachweis von Immunglobulin G (IgG)-Antikörpern im Blutserum, die in der Regel in niedrigem Titer zirkulieren und sich an die apikale Oberfläche der Enterozyten binden (Abb. 2). In einem Bericht über 25 Kinder mit chronischer unspezifischer Diarrhoe fanden sich bei 14 (56\%) der Patienten Enterozyten-Autoantikörper im Vergleich zu $3(5 \%)$ von 53 Patienten mit chronischer entzündlicher Darmerkrankung, 0 von 10 Zöliakiepatienten und 0 von 50 Kindern mit anderen, nicht mit dem Gastrointestinaltrakt in Zusammenhang stehenden Erkrankungen [16]. Es handelt sich hierbei nicht um einen Antikörper, der für ein Epitop der humanen Enterozyten spezifisch ist, da sich die Antikörper auch an die Dünndarmepithelzellen von Schnittpräparaten binden, die aus 
dem Darmgewebe von Ratten, Mäusen und Meerschweinchen gewonnen wurden. Das Antigen findet sich ferner auch im Nierenepithel.

Die Westernblots zeigen, dass Autoantigene ein Molekulargewicht von entweder 55 [17] oder 75 kDA [18, 19] haben. Das 75 kDA grosse Autoantigen wird im Dünndarm, im Dickdarm, in der Bauchspeicheldrüse und in der Niere exprimiert [20]. Bei der Behandlung (nähere Ausführungen unten) werden die Titer der zirkulierenden Enterozyten-Autoantikörper gesenkt bzw. allmählich aber vollständig abgebaut, wobei sich der klinische $\mathrm{Zu}-$ stand verbessert. Es bietet sich daher an, die Blutspiegel der systemischen Autoantikörper zu beobachten, die ein objektiver Massstab dafür sind, ob eine klinische Intervention anspricht oder nicht [17].

Zwar wurden Zielproteine für Enterozyten-Autoantikörper beschrieben, der Test ist jedoch laut anderen Forschern möglicherweise nicht ausreichend genau. MartinVilla et al. [21] beispielsweise wiesen diese Autoantikörper im systemischen Kreislauf von Patienten nach, bei denen weder eine Zottenatrophie noch in der Anamnese ein Hinweis auf Diarrhoe festzustellen war. Diese Untersuchung auf Enterozyten-Autoantikörper muss folglich wie alle anderen Screening-Tests auch in dem entsprechenden klinisch-pathologischen Kontext erfolgen.

Einige Fallberichte beschreiben eine Autoimmun-Enteropathie, bei der sich die systemischen Autoantikörper eher gegen die Becherzellen als gegen die apikalen Regionen der Enterozyten richten [22]. Eine Darmbiopsie zeigt in diesen Fällen einen Mangel an Becher-, Panethund enteroendokrinen Zellen [23]. Dies spiegelt möglicherweise einen Mangel an Transkriptionsfaktor Math1 wider [24]. Ansonsten ähneln die klinischen und pathologischen Erkenntnisse den Ergebnissen anderer Fälle der Autoimmun-Enteropathie. Dieses Ergebnis bekräftigt die Ansicht, dass infiltrierende T-Zellen bei der Pathobiologie der Autoimmun-Enteropathie eher eine Rolle spielen als systemische Autoantikörper. Die Autoantikörper sind daher wahrscheinlich eher Marker für eine zugrunde liegende Erkrankung, die infolge der Entzündung und Läsionen der Darmschleimhaut entsteht, und sind nicht speziell an der Pathogenese der Erkrankung beteiligt [13, 14].

\section{Primäre Immundefekte}

Intestinale Läsionen, die Enteropathie, Malabsorption von Nährstoffen und protrahierte Diarrhoe zur Folge haben, können durch eine Reihe immunvermittelter
Krankheiten genetischen Ursprungs verursacht werden [25]. So werden beispielsweise sowohl die chronische Granulomatose als auch die Glycogenose (Glycogenspeicherkrankheit) Typ 1b mit einer Entzündung der Darmschleimhaut im gesamten Gastrointestinaltrakt in Verbindung gebracht, wobei die gleichen klinischen, radiologischen und endoskopischen Merkmale beobachtet werden wie bei der chronisch-entzündlichen Darmerkrankung. Auch die eosinophile Gastroenteritis sollte bei der Differenzialdiagnose in Betracht gezogen werden.

Inzwischen ist klar, dass der früher pauschal als $\mathrm{Au}-$ toimmun-Enteropathie bezeichneten Erkrankung multiple Ursachen zugrunde liegen. So gibt es beispielsweise Berichte darüber, dass sich primäre Immundefekte, welche die T-Zellen betreffen, mit den Symptomen einer Autoimmunerkrankung manifestieren. Arniaz-Villena et al. [26] berichteten beispielsweise über eine Familie, in der ein kongenitaler Defekt der Expression der CD3$\gamma$-Untereinheit des T-Zell-Rezeptorkomplexes vorlag. Bei dem betroffenen Patienten wurde eine protrahierte Diarrhoe in Zusammenhang mit Autoantikörpern gegen Darmepithelzellen, glatte Muskelzellen und Mitochondrien sowie ferner eine autoimmune hämolytische Anämie und ein selektiver Mangel des IgG-Isotypen IgG2 festgestellt.

In der Literatur findet sich ferner die Beschreibung zweier Kinder mit immuno-ossärer Dysplasie Schimke (IODS) und abnormer Immunfunktion der T-Zellen [27, 28]. Beide Kinder litten unter chronischer Diarrhoe und Symptomen, die mit einer Autoimmun-Enteropathie vereinbar sind. Es konnten jedoch keine Autoantikörper nachgewiesen werden und bei einem der beiden Kinder wurden in der Dünndarmmucosa atypische Myobakterien festgestellt [28]. Diese Beobachtung untermauert und hebt nochmals hervor, wie wichtig es ist, bei Kindern mit chronischer unspezifischer Diarrhoe chronische Infektionen sowie einen zugrunde liegenden Immundefekt auszuschliessen.

\section{IPEX-Syndrom}

Das X-chromosomal vererbte Syndrom mit Immundysregulation, Polyendokrinopathie und Enteropathie (IPEX) ist die spezifischste und am besten dokumentierte Ursache der Autoimmun-Enteropathie. Die Krankheit wird durch Mutationen des FOXP3-Gens verursacht, das sich in der Perizentromer-Region des X-Chromosoms befindet: Xp1 1.23-Xp13.3 [29, 30]. FOXP3 (häufig auch als 
Abb. 3. Die histologische Untersuchung des Dünndarms eines Kindes mit IPEXSyndrom belegt eine totale Zottenatrophie sowie eine ausgeprägte Entzündung der Lamina propria mit chronisch-endzündlichem Zellinfiltrat.

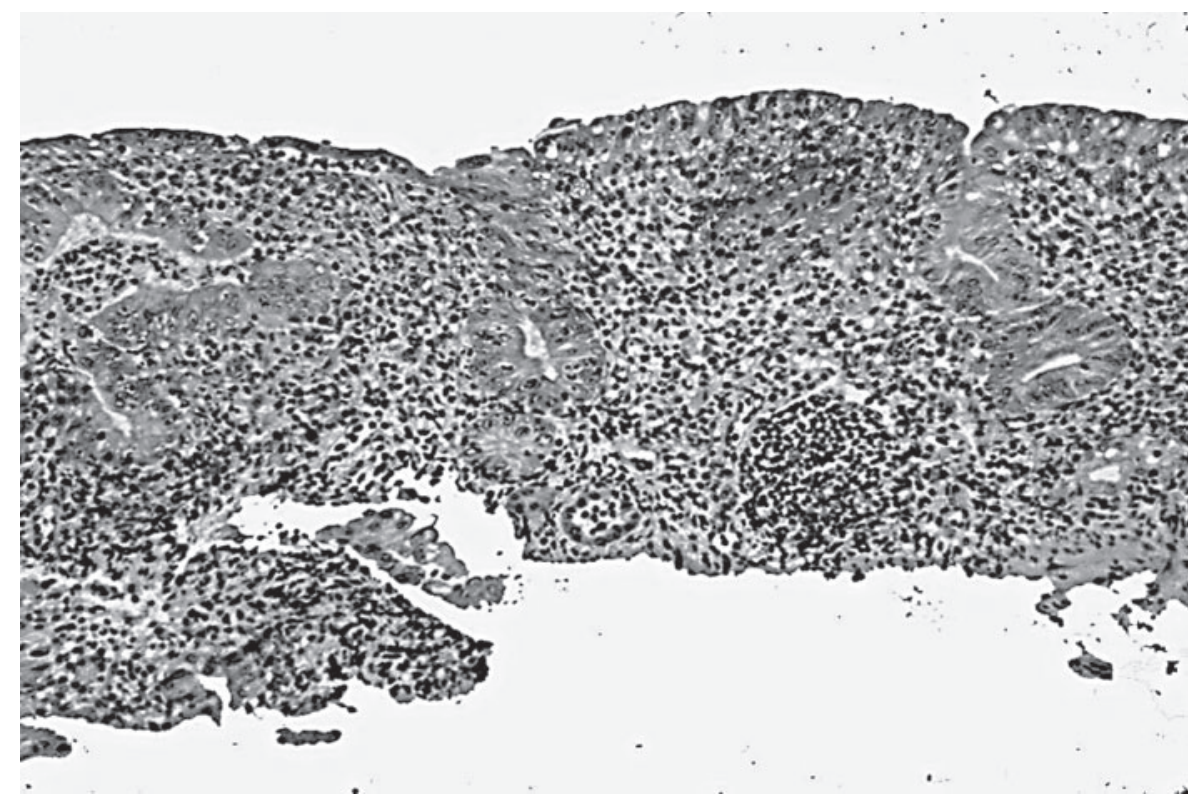

JM2 und Scurfin bezeichnet) ist ein Transkriptionsfaktor, der für die Steuerung der normalen Bildung von regulatorischen T-Zellen wesentlich ist [31, 32]. Eine Mutation dieses Transkriptionsfaktors führt zum Wegfall der normalen Steuerung der CD25+ T-Zellen [33, 34] und zu einer unangemessenen Aktivierung sowie Streuung der aktivierten Immunzellen auf multiple Organe, u.a. den Intestinaltrakt (Abb. 3).

Es wurden mehrere Mutationen des FOXP3-Gens beschrieben, die Krankheiten beim Menschen verursachen. Die bezüglich des klinischen Bilds, des Schweregrads der Krankheit, der Anzahl der autoimmunen Manifestationen ausserhalb des Verdauungstrakts und der Prognosen beobachteten Unterschiede lassen sich wahrscheinlich durch eine Genotyp-Phänotyp-Beziehung erklären [35]. Bei der Autoimmun-Enteropathie kann demnach folgende Unterscheidung vorgenommen werden: Fälle, die durch IPEX verursacht werden, Patienten männlichen Geschlechts, bei denen keine Mutation des FOXP3-Gens nachgewiesen werden kann und Patienten weiblichen Geschlechts [36].

Säuglinge und Kleinkinder männlichen Geschlechts mit IPEX-Syndrom weisen Typ-1-Diabetes mellitus, Autoimmun-Thyroiditis, eine autoimmun-hämolytische Anämie (positiver direkter Coombs-Test) und diverse Hauterkrankungen auf, u.a. Ekzem, ichthyosiforme Erythrodermie, Psoriasis und Alopecia universalis [37]. $\mathrm{Zu}$ den markanten Merkmalen zählt in der Regel eine durch Autoimmun-Enteropathie verursachte, protrahierte voluminöse Diarrhoe. Kinder mit IPEX-Syndrom weisen möglicherweise auch atopische Symptome auf, u.a. Nahrungsmittelallergien, periphere Eosinophilie und erhöhte Immunglobulin E (IgE)-Spiegel [38]. Bei Jungen mit Autoimmun-Enteropathie (insbesondere in Fällen, in denen auch weitere Geschwister betroffen sind), bei denen sich Autoantikörper, die sich gegen andere Organe richten, und systemische Autoimmunerkrankungen, die bereits in der medizinischen Literatur beschrieben wurden, nachweisen lassen, sollte das IPEXSyndrom bei der Diagnose als Möglichkeit in Betracht gezogen werden.

\section{Therapeutische Überlegungen}

Die Anfangsbehandlung ist bei Säuglingen und Kleinkindern mit chronischer Diarrhoe und Verdacht auf Autoimmun-Enteropathie genau die gleiche wie bei allen anderen Patienten mit protrahierter Diarrhoe. Die Behandlung sollte sich primär auf die Aufrechterhaltung des intravaskulären Volumens und auf die Ernährungsrehabilitation konzentrieren, was im Allgemeinen eine kontinuierliche Elementardiät entweder durch enterale Ernährung oder durch TPE (totale parenterale Ernährung) erfordert. Häufig wird bei den Kindern im Krankenhaus nichts unternommen, um den Zustand der Dehydrierung 
und Mangelernährung zu beheben, bis die Ergebnisse von umfassenden diagnostischen Bewertungen vorliegen. Dieser Ansatz ist bedauernswert: die Versorgung mit Flüssigkeit und Nährstoffen zählt zu den wichtigsten Prioritäten [39]. Erst nachdem diese Versorgung erfolgt ist, sollten die Ärzte innehalten und sich bei der Differenzialdiagnose durch die lange Liste der Möglichkeiten arbeiten.

Die Autoimmun-Enteropathie spricht in der Regel gut auf eine starke Suppression des wirtseigenen Immunsystems an. Mit Hilfe hochdosierter systemischer Kortikosteroide kann die Schleimhauentzündung häufig verringert, die Stuhlmenge gesenkt und somit die enterale Kalorienzufuhr gesteigert werden.

Die Autoantikörper sinken parallel zu dem klinischen Ansprechen des Patienten auf die medizinische Behandlung. Eine Steroidabhängigkeit (d.h. ein erneutes Auftreten der Symptome nach langsamem Ausschleichen der Kortikosteroiddosis) ist die Regel. Auch eine Steroidresistenz (d.h. das Ausbleiben eines augenscheinlichen Ansprechens auf die Therapie) kommt häufiger vor. Folglich hat man eine Reihe weiterer starker Immunsuppressiva eingesetzt. Fallberichte deuten bei einer Reihe verschiedener Therapien, wie z.B. Cyclosporin [40, 41], Tacrolimus [42], Sirolimus [43], Mycophenolat Mofetil [44], Cyclophosphamid [45] und biologischer Agenzien, wie z.B. dem rekombinanten monoklonalen Tumornekrosefaktor- $\alpha$-Antikörper Infliximab, eine nützliche Wirkung an [46].

Die meisten der betroffenen Kinder sprechen auf eine starke Suppression des Immunsystems an und vertragen mit der Zeit eine Zunahme der enteralen Ernährung bei gleichzeitiger Reduzierung der supportiven parenteralen Ernährung. Eine Autoimmun-Enteropathie sollte daher nicht als Indikation für eine Dünndarmtransplantation gesehen werden. Diese klinische Prognose unterscheidet sich stark von den meisten Fällen der Mikrovillusatrophie, bei denen die Patienten nicht auf eine starke Immunsuppression ansprechen und deren Outcome unsicher ist, wird nicht die Dünndarmtransplantation zumindest als eine therapeutische Option in Erwägung gezogen $[1,6,12]$.

Ob diese Kinder möglicherweise von alternativen therapeutischen Strategien profitieren, beispielsweise einer Knochenmarktransplantation, ist nicht bekannt. Die durch die Transplantation von Knochenmarkzellen bei Kindern mit IPEX-Syndrom [47] gemeldeten nützlichen Wirkungen lassen darauf schliessen, dass die nähere Erwägung dieses Verfahrens bei schweren Fällen, die nur eingeschränkt bzw. teilweise auf eine starke Suppression des Immunsystems ansprechen, lohnend ist. Die positiven Reaktionen von Kindern mit IPEX-Syndrom auf allogene Knochenmarktransplantationen unterstreichen ferner, dass die richtige Diagnose der zugrunde liegenden Krankheit bei der frühkindlichen protrahierten Diarrhoe von entscheidender Bedeutung ist. Die Pauschaldiagnose «frühkindliche protrahierte therapieresistente Diarrhoe» ist in diesen Fällen nicht hilfreich und erweist sich bei der Erwägung der geeigneten Behandlungsoptionen möglicherweise als hinderlich.

Auch bei der Erwägung einer genetischen Beratung für Familien mit betroffenen Kindern ist eine sorgfältige Prüfung der genauen Ätiologie der protrahierten Diarrhoe wesentlich. Die meisten Fälle der Autoimmun-Enteropathie scheinen sporadisch aufzutreten, d.h. es sind keine weiteren Familienmitglieder betroffen, und es lässt sich auch keine Konsanguinität der Eltern feststellen. Andererseits handelt es sich bei dem IPEX-Syndrom um eine X-chromosomale Erkrankung. Dieser Umstand ruft weitere Fragen hinsichtlich des Risikos für die betroffenen Familien hervor, die jedoch den Rahmen dieses Artikels sprengen würden.

\section{Schlussfolgerungen}

Der Begriff Autoimmun-Enteropathie bezeichnet eine relativ seltene Ursache der frühkindlichen protrahierten Diarrhoe. Es handelt sich hierbei um eine schwere Entzündung, die in der Regel auf eine Behandlung mit Immunsuppressiva anspricht. Aller Wahrscheinlichkeit nach umfasst die Autoimmun-Enteropathie eine Reihe von Ätiologien, die besser definiert werden müssen, möchte man zukünftig gezielt geeignete Therapien und genetische Beratung bereitstellen. Die zugrunde liegenden Immundefekte und pathophysiologischen Prozesse, welche die klinischen Manifestationen der Erkrankung verursachen, sollten durch weitere Forschungsarbeiten bestimmt werden. Entsprechende relevante Tiermodelle werden sich bei der Beschreibung der Krankheitsprozesse als äusserst hilfreich erweisen.

Zur Identifikation der für die Autoimmun-Enteropathie verantwortlichen genetischen Mutationen sollten die aus einer Hautbiopsie gewonnenen Fibroblasten für eine Zellkultur immortalisiert werden. Ebenso sollte aus Darmbiopsien die Boten-RNA (mRNA, messenger RNA) isoliert und bei einem kompletten Genom-Scanning, einer technisch bereits machbaren und wirtschaftlich sinnvollen Methode, die ein aussagekräftiges Forschungstool darstellt, analysiert werden [48]. Ein ge- 
meinsames internationales Forschungsprojekt wird darüber hinaus die Einblicke in die molekulare Basis chronischer immunvermittelter Enteropathien verbessern.

\section{Danksagung}

P.M.S. hält einen Lehrstuhl im Forschungsbereich Gastrointestinale Erkrankungen in Kanada. Die in den Laboratorien der Verfasser durchgeführten Tätigkeiten werden durch Beihilfen des kanadischen Institute of Health Research finanziert.

\section{Literatur}

1 Sherman PM, Mitchell DJ, Cutz E: Neonatal enteropathies: defining the causes of protracted diarrhea of infancy. J Pediatr Gastroenterol Nutr 2004;38:16-26

2 Camilleri M: Chronic diarrhea: a review on pathophysiology and management for the clinical gastroenterologist. Clin Gastroenterol Hepatol 2004;2:198-206.

3 Schiller LR: Chronic diarrhea. Gastroenterology 2004;127:287-293.

4 Bhutta ZA, Ghishan F, Lindley K, Memon IA, Mittal S, Rhoads JM: Persistent and chronic diarrhea and malabsorption: Working Group report of the second World Congress of Pediatric Gastroenterology, Hepatology, and $\mathrm{Nu}-$ trition. J Pediatr Gastroenterol Nutr 2004 39(suppl 2):S711-S716

5 Hill ID, Dirks MH, Liptak GS, et al: Guideline for the diagnosis and treatment of celiac disease in children: recommendations of the North American Society for Pediatric Gastroenterology, Hepatology and Nutrition. J Pediatr Gastroenterol Nutr 2005;40:1-19.

6 Cutz E, Rhoads JM, Drumm B, et al: Microvillus inclusion disease: an inherited defect of brush-border assembly and differentiation. $\mathrm{N}$ Engl J Med 1989;320:646-651.

7 Phillips AD, Schmitz J: Familial microvillous atrophy: a clinicopathological survey of 23 cases. J Pediatr Gastroenterol Nutr 1992;14: 380-396.

-8 Reifen RM, Cutz E, Griffiths AM, et al: Tufting enteropathy: a newly recognized clinicopathological entity associated with refractory diarrhea in infants. J Pediatr Gastroenterol Nutr 1994; 18:379-385.

9 Patey N, Scoazec JY, Cuenod-Jabri B, et al: Distribution of cell adhesion molecules in infants with intestinal epithelial dysplasia (tufting enteropathy). Gastroenterology 1997;113: 833-843.

10 Pirisi-Hauck NC, Foss H-D, Baier J, Kurunczi S: Simultaneous occurrence of autoimmune enteropathy and recurrent deep vein thrombosis. J Pediatr Gastroenterol Nutr 2000;30:324329.

11 Carroccio A, Volta U, Di Prima L, et al: Autoimmune enteropathy and colitis in an adult patient. Dig Dis Sci 2003;48:1600-1606.

12 Cutz E, Sherman PM, Davidson GP: Enteropathies associated with protracted diarrhea of infancy: clinicopathological features, cellular and molecular mechanisms. Pediatr Pathol Lab Med 1997;17:335-368.
13 Murch SH, Fertleman CR, Rodrigues C, et al: Autoimmune enteropathy with distinct mucosal features in T-cell activation deficiency: the contribution of $\mathrm{T}$ cells to the mucosal lesion. $\mathrm{J}$ Pediatr Gastroenterol Nutr 1999;28:393399.

14 Cuenod B, Brousse N, Goulet O, et al: Classification of intractable diarrhea of infancy using clinical and immunohistological criteria. Gastroenterology 1990;99:1037-1043.

5 King C, Lic A, Koelsch K, Sarvetnick N: Homeostatic expansion of $\mathrm{T}$ cells during immune insufficiency generates autoimmunity. Cell 2004:117:265-277.

16 Mirakian R, Richardson A, Milla PJ, et al: Protracted diarrhoea of infancy: evidence in support of an autoimmune variant. Br Med $\mathrm{J}$ (Clin Res Ed) 1986;293:1132-1136.

17 Colletti RB, Guillot AP, Rosen S, et al: Autoimmune enteropathy and nephropathy with circulating anti-epithelial cell antibodies. J Pediatr 1991;118:858-864

18 Kobayashi I, Imamura K, Yamada M, et al: A 75-kD autoantigen recognized by sera from patients with X-linked autoimmune enteropathy associated with nephropathy. Clin Exp Immunol 1998;111:527-531.

19 Kobayashi I, Imamura K, Kubota M, et al: Identification of an autoimmune enteropathyrelated 75-kilodalton antigen. Gastroenterology 1999;117:823-830.

20 Ishikawa S, Kobayashi I, Hamada J-I, et al: Interaction of MCC2, a novel homologue of MCC tumor suppressor, with PDZ-domain protein AIE-75. Gene 2001;267:101-110.

21 Martin-Villa JM, Regueiro JR, De Juan D, et al: T-lymphocyte dysfunctions occurring together with apical gut epithelial cell autoantibodies. Gastroenterology 1991;101:390-397.

22 Moore L, Xu X, Davidson G, et al: Autoimmune enteropathy with anti-goblet cell antibodies. Hum Pathol 1995;26:1162-1168.

23 Shaoul R, Hong D, Okada Y, Cutz E, Marcon MA: Lineage development in a patient without goblet, Paneth and enteroendocrine cells: a clue for intestinal epithelial differentiation. Pediatr Res 2005;58:492-498.

24 Yang Q, Berminham A, Finegold MJ, et al: Requirement of Math1 for secretory cell lineage commitment in the mouse intestine. Science 2001;294:2155-2158
25 Goulet O, Seidman E: Gastrointestinal manifestations of immunodeficiency. 1. Primary immunodeficiency diseases; in Walker W, Goulet O, Kleinman R, Sherman P, Shneider B, Sanderson I (eds): Pediatric Gastrointestinal Disease, ed 4. Philadelphia, B.C. Decker, 2004, pp 707-733.

26 Arniaz-Villena A, Timon M, Corell A, et al: Primary immunodeficiency caused by mutations in the gene encoding the CD3-gamma subunit of the T-lymphocyte receptor. N Engl J Med 1992;327:529-533.

27 Kaitila I, Savilahti E, Ormala T: Autoimmune enteropathy in Schimke immunoosseous dysplasia. Am J Med Genet 1998;77:427-430.

28 Stormon M, Friedman J, King S, Cutz E, Furuya $\mathrm{KN}$ : An unusual case of diarrhea in Schimke immuno-osseous dysplasia. J Pediatr Gastroenterol Nutr 2002;35:369-371

29 Bennett CL, Christie J, Ramsdell F, et al: The immune dysregulation, polyendocrinopathy, enteropathy, X-linked syndrome (IPEX) is caused by mutations of FOXP3. Nat Genet 2001:27:20-21.

-30 Ochs HD, Ziegler SF, Torgerson TR: FOXP3 acts as a rheostat of the immune response. Immunol Rev 2005;203:156-164.

31 Hori S, Nomura T, Sakaguchi S: Control of regulatory $\mathrm{T}$ cell development by the transcription factor FOXP3. Science 2003;299:10571061.

- 32 Hori S, Sakaguchi S: Foxp3: a critical regulator of the development and function of regulatory T cells. Microbes Infect 2004;6:745-751.

- 33 Fontenot JD, Gavin MA, Rudensky AY: FOXP3 programs the development and function of CD4+CD25+ regulatory $\mathrm{T}$ cells. Nat Immunol 2003;4:330-336.

34 Bakke AC, Purtzer MZ, Wildin RS: Prospective immunological profiling in a case of immune dysregulation, polyendocrinopathy, enteropathy, X-linked syndrome (IPEX). Clin Exp Immunol 2004;137:373-378.

35 Eisenbarth GS, Gottlieb PA: Autoimmune polyendocrine syndromes. N Engl J Med 2004; 350:2068-2079.

36 Ruemmele FM, Brousse N, Goulet O: Autoimmune enteropathy: molecular concepts. Curr Opin Gastroenterol 2004;20:587-591.

-37 Nieves DS, Phipps RP, Pollock SJ, et al: Dermatologic and immunologic findings in the immune dysregulation, polyendocrinopathy, enteropathy, X-linked syndrome. Arch Dermatol 2004; 140:466-472. 
38 Patel DD: Escape from tolerance in the human $\mathrm{X}$-linked autoimmunity-allergic disregulation syndrome and the Scurfy mouse. J Clin Invest 2001;107:155-157.

-39 Lo CW, Walker WA: Chronic protracted diarrhea of infancy: a nutritional disease. Pediatrics 1983; 72:786-800.

-40 Seidman EG, Lacaille F, Russo P, et al: Successful treatment of autoimmune enteropathy with cyclosporine. J Pediatr 1990;117:929932.

41 Sanderson IR, Phillips AD, Spencer J, WalkerSmith JA: Response to autoimmune enteropathy to cyclosporin A therapy. Gut 1991;32: 1421-1425.
42 Bousvaros A, Leichtner AM, Book L, et al: Treatment of pediatric autoimmune enteropathy with tacrolimus (FK506). Gastroenterology 1996;111:237-243.

43 Bindl L, Torgerson T, Perroni L, Youssef N, Ochs HD, Goulet O, Ruemmele FM: Successful use of the new immune-suppressor sirolimus in IPEX (immune dysregulation, polyendocrinopathy, enteropathy, X-linked syndrome). J Pediatr 2005;147:256-259.

44 Quiroz-Tejeira RE, Ament ME, Vargas JH: Induction of remission in a child with autoimmune enteropathy using mycophenolate mofetil. J Pediatr Gastroenterol Nutr 2003; 36:482-485.
45 Oliva-Hemper MM, Loeb DM, Abraham SC, et al: Remission of severe autoimmune enteropathy after treatment with high-dose cyclophosphamide. J Pediatr Gastroenterol Nutr 2003;36:639-643.

46 Vanderhoof JA, Young RJ: Autoimmune enteropathy in a child: response to infliximab therapy. J Pediatr Gastroenterol Nutr 2002;34: 312-316.

47 Baud O, Goulet O, Canioni D, et al: Treatment of the immune dysregulation, polyendocrinopathy, enteropathy, X-linked sydrome (IPEX) by allogeneic bone marrow transplantation. $\mathrm{N}$ Engl J Med 2001;344:1758-1762.

48 Collins SM, McHugh K, Croitoru K, et al: The establishment of a national tissue bank for inflammatory bowel disease research in Canada. Can J Gastroenterol 2003;17:107-109. 